\title{
Treatment outcomes of civilian gunshot tibia fractures at a major trauma centre
}

\section{Muaad Gerafa, Shafique Jakoet, Marcus van Heukelum, Nicholas le Roux, Simone van der Merwe, Obakeng Makhubalo, Gian du Preez, Marilize Burger, Nando Ferreira*(i)}

Division of Orthopaedic Surgery, Department of Surgical Sciences, Faculty of Medicine and Health Sciences, Stellenbosch University, Cape Town, South Africa

${ }^{*}$ Corresponding author: drferreiran@gmail.com

Citation: Gerafa M, Jakoet S, Van Heukelum M, Le Roux N, Van der Merwe S, Makhubalo O, Du Preez G, Burger M, Ferreira N. Treatment outcomes of civilian gunshot tibia fractures at a major trauma centre. SA Orthop J 2021;20(2):71-75. http://dx.doi. org/10.17159/2309-8309/2021/ v20n2a1

Editor: Prof. Leonard C Marais, University of KwaZulu-Natal, Durban, South Africa

Received: July 2020

Accepted: November 2020

Published: May 2021

Copyright: @ 2021 Gerafa M. This is an open-access article distributed under the terms of the Creative Commons

Attribution Licence, which permits unrestricted use, distribution and reproduction in any medium, provided the original author and source are credited.

Funding: No funding was received for this study.

Conflict of interest: The authors declare they have no conflicts of interest that are directly or indirectly related to this research.

\begin{abstract}
\section{Background}

The aim of this retrospective longitudinal study was to describe the overall burden and outcomes of surgically managed gunshot tibia fractures at a major trauma centre. Secondary objectives were to identify possible risk factors for complications including non-union and infection and to highlight any differences in outcomes between treatment modalities.
\end{abstract}

\section{Methods}

All consecutive patients who sustained gunshot injuries to the tibia between January 2014 and December 2017 including children and multiple gunshots injuries were considered for inclusion. Information related to patient demographics, injury characteristics, treatment information and treatment outcomes with respect to rate of fracture union and occurrence of infection were obtained from patient records. All patients with insufficient medical records were excluded.

\section{Results}

The records of 197 patients who sustained gunshot tibia fractures were reviewed. The mean follow-up was 4.1 months (interquartile range [IQR] 2.5-6.8). The majority of cases were young males $(89 \%)$ with a mean age of $29.2 \pm 10.2$ years. Extra-articular diaphyseal fractures were observed in the majority of cases $(91 \%)$. Definitive treatment included formal debridement in theatre and plaster cast immobilisation (44\%), intramedullary nail fixation $(27 \%)$, circular external fixation $(22 \%)$ and plate fixation $(7 \%)$. The study revealed an overall fracture-related infection (FRI) rate of $11 \%$ and bone union rate of $91 \%$. Circular external fixation showed the lowest fracture union rate $(86 \%)$ and highest FRI rate $(21 \%)$ of the modalities included in this study. No associations between independent risk factors and presence of complications were identified.

\section{Conclusion}

The study reports encouraging outcomes for tibia fractures caused by civilian gunshot injuries. Various definitive surgical stabilisation techniques showed high proportions of union and low burden of FRI.

Level of evidence: Level 4

Keywords: tibia, gunshot, fracture, outcome

\section{Introduction}

Trauma accounted for $8.5 \%$ of global deaths as indicated in the 2015 Global Burden of Disease study. ${ }^{1}$ Trauma, specifically, can be classified into intentional injuries that occurred due to interpersonal or collective violence and self-inflicted injury, as well as unintentional injuries including motor vehicle collisions, falls and burns. The United Nations Office on Drugs and Crime (UNODC) has ranked South Africa (SA) 11th in terms of violence, with a homicide rate of 31.1 per 100000 people. $^{2}$ A 2019 study by Zaidi et al. highlighted the high monthly load of trauma at a district hospital in the Western Cape province of South Africa in which $44.4 \%$ of trauma cases were attributed to interpersonal violence, where $7.2 \%$ of these were firearm-related. ${ }^{3}$
Insight into the South African burden of gunshot-related injuries on orthopaedic services was reported by Engelmann et al. who reviewed 294 extremity gunshot injuries seen at a tertiary trauma centre in Cape Town over an 11-month period between June 2015 and April 2016, and reported that tibia fractures accounted for the majority $(42.2 \%)$ of lower extremity injuries. ${ }^{4} \mathrm{~A}$ retrospective study by Martin et al. reviewed 111 patients at a tertiary level hospital who had sustained 147 gunshot-induced fractures over a 12-month period. The authors reported that the majority of these injuries involved the lower extremity, and $95 \%$ of these patients inflicted with lower extremity gunshot-induced fractures required orthopaedic procedures at an average cost exceeding 13 times the annual average per patient afforded by the South African public health sector. ${ }^{5}$ In agreement with this, a recent study from a tertiary 
hospital in South Africa reported a total of 1449 gunshot-related orthopaedic fractures over a four-year period, resulting in a total cost of more than ZAR 50 million to the healthcare system. ${ }^{6}$

Gunshot-induced tibia fractures are often associated with a myriad of complications presenting greater challenges to clinicians. Gunshots involve the transfer of blast energy to the bone and soft tissue resulting in cavitary tissue damage and a large zone of injury. ${ }^{8}$ Further, the risk of infection, compartment syndrome, nerve palsies, delayed union or non-union are increased due to the subcutaneous location of the tibia. Moreover, poor correlation between external wound size and degree of underlying tissue damage may lead to an underestimation of the injury severity. ${ }^{8}$

Several controversies regarding the management of gunshotinduced tibia fractures persist as a result of the heterogeneity of these injuries. Management of these injuries can range from conservative management to formal debridement and skeletal stabilisation either in a single setting, or as a staged approach. ${ }^{9}$ Advantages of temporary monolateral external fixation include bone stabilisation, and easy access for wound care. ${ }^{10}$ Conversely, problems associated with monolateral external fixation include pin-site infection and loss of stability during long-term use. ${ }^{10}$ In comparison, studies have reported the efficacy of formal debridement and internal fixation in the same sitting, demonstrating the advantages such as access to soft tissue and low risk of deep infection. ${ }^{11}$ These reports add to the controversy regarding the management of gunshot-induced fractures and highlight the lack of evidence-based treatment guidelines.

The present study aims to describe the treatment outcomes of civilian gunshot tibia fractures at a major trauma centre. ${ }^{12}$ Secondary objectives were to identify possible risk factors for complications, including non-union and fracture-related infection (FRI).

\section{Methods}

A retrospective review of clinical records and serial radiographs of all patients who sustained gunshot tibia fractures between January 2014 and December 2017 was conducted. Institutional ethics committee approval and hospital board approval was obtained prior to commencement of data collection.

Medical records of all adult and paediatric patients presenting with tibia fractures as a result of gunshot injuries in the study period were reviewed by the authors. Demographic information of all included patients was recorded, and patient records were assessed to identify the extent of soft tissue injury and presence of associated injuries. Patients that did not require surgical intervention, as well as all patients with incomplete medical records, were excluded. Tibia fractures were classified according to the Muller AO Classification ${ }^{13}$ as intra-articular (AO 41B-C and 43B-C), metaphyseal (AO 41A and 43A) and diaphyseal (AO 42A-C) for analysis purposes.

As per international protocol, intravenous antibiotics (cephazolin) were administered on presentation ${ }^{14}$ and the treatment modality used, as well as the timing thereof, was recorded. Temporary external fixation was used for damage control and patients with vascular injuries. For definitive management, interlocked intramedullary nailing (IMN) was used for diaphyseal tibia fractures where the soft tissue envelope was amenable to primary closure, while intra-articular and metaphyseal fractures were most often managed with plate fixation. Fine wire circular external fixation was used for metaphyseal and intra-articular fractures with extensive soft tissue damage or where delays to surgery were encountered, due to the trauma burden at our institution.

Radiographs were reviewed to determine time to radiological union based on the Radiographic Union Score for Tibia fractures
(RUST Score). ${ }^{15}$ The presence of an FRI was deemed 'suggestive' or 'confirmed' using the guidelines outlined in the consensus document of Metsemakers et al. ${ }^{16}$ Minor complications, such as the presence of cast-associated pressure sores or pin-site infections, were not recorded.

Data was analysed using STATISTICA version 13.5 (StatSoft Inc., Tulsa, OK, USA) and data is presented as means \pm standard deviations or median (interquartile range [IQR]), depending on the distribution, while categorical data is presented as frequencies and counts. Associations between risk factors, including age, sex, HIV status, injury location and delay to surgical management, and known outcomes, specifically presence of i) non-union or ii) infection, were investigated using an independent t-test or Mann-Whitney $U$ test for continuous data, while a Pearson's chisquare test or a Fisher's exact test was used for categorical data.

Table I: Demographic information of included patients

\begin{tabular}{|l|c|}
\hline Age (years) & $\mathrm{n}=197$ \\
\hline Sex & $(28.8 \pm 10.4)(197)$ \\
\hline Male & $89 \%(175)$ \\
\hline Female & $11 \%(22)$ \\
\hline HIV status & \\
\hline Positive & $34 \%(67)$ \\
\hline Negative & $28 \%(56)$ \\
\hline Unknown & $38 \%(74)$ \\
\hline
\end{tabular}

Data is expressed as means \pm standard deviations or as frequencies, with counts indicated in parentheses.

Table II: Injury characteristics of included patients

\begin{tabular}{|c|c|}
\hline & $n=197$ \\
\hline \multicolumn{2}{|l|}{ Affected side } \\
\hline Left & $50 \%(99)$ \\
\hline Right & $50 \%(98)$ \\
\hline \multicolumn{2}{|l|}{ Anatomical site } \\
\hline Tibia & $96 \%(189)$ \\
\hline Tibia and fibula & $4 \%(7)$ \\
\hline Tibia and patella & $0.5 \%(1)$ \\
\hline \multicolumn{2}{|l|}{ Intra-articular } \\
\hline No & $95 \%(187)$ \\
\hline Yes & $5 \%(10)$ \\
\hline \multicolumn{2}{|c|}{ AO classification (tibia fracture) } \\
\hline Diaphysis & $91 \%(179)$ \\
\hline 42B3 & $71 \%(140)$ \\
\hline $42 \mathrm{~A} 3$ & $15 \%(30)$ \\
\hline $42 \mathrm{~B} 2$ & $5 \%(9)$ \\
\hline Proximal metaphysis & $8 \%(16)$ \\
\hline $41 \mathrm{C} 2$ & $4 \%(8)$ \\
\hline $41 \mathrm{~A} 3$ & $4 \%(8)$ \\
\hline Distal metaphysis & $1 \%(2)$ \\
\hline $43 C 2$ & $0.5 \%(1)$ \\
\hline $43 C 3$ & $0.5 \%(1)$ \\
\hline
\end{tabular}

Data is expressed as frequencies with counts in parentheses. 


\section{Results}

A total of 213 patients who sustained gunshot tibia injuries were reviewed. Sixteen patients who did not require surgery or had incomplete medical records were excluded. The final cohort therefore included 175 male patients $(89 \%)$ and 22 female patients $(11 \%)$ with a mean age of $28.8 \pm 10.4$ years (Table $I$ ). Isolated tibial injures represented $96 \%$ of cases $(n=189)$. The majority of injuries $(91 \%, n=179)$ were diaphyseal fractures while $9 \% \quad(n=18)$ were peri-articular injuries (Table II).

Patients underwent surgery at a median of 2.0 (IQR 2.0-5.0) days following admission. The majority of fractures were managed by fixation including IMN fixation $(27 \%, n=53)$, circulator external fixation $(22 \%, n=44)$, and plate fixation $(7 \%, n=14)$ while remaining fractures were managed through formal debridement and plaster cast immobilisation $(44 \%, n=86)$. Definitive soft tissue and fracture management was performed during a single theatre visit in all patients. Median length of stay in hospital was 6.0 (IQR 4.0-9.0) days. Patients were followed up for a median of 4.1 (IQR 2.56.8) months. Five $(3 \%)$ patients were lost to follow-up prior to confirmation of union (Table III).

Table III: Treatment information of included patients

\begin{tabular}{|l|c|}
\hline Time delay (admission to surgery) (days) & $\mathbf{n = 1 9 7}$ \\
\hline Admitted to hospital & $2(2-5)(186)$ \\
\hline Admitted to ICU & $100 \%(197)$ \\
\hline $\begin{array}{l}\text { Temporary external fixator } \\
\text { Definitive procedure }\end{array}$ & $3 \%(6)$ \\
\hline \multicolumn{1}{|c|}{ Debridement and plaster cast } \\
$\quad$ Intramedullary nail \\
$\quad$ Circular external fixator \\
\hline \multicolumn{1}{|c|}{ Plate fixation } \\
\hline Hospital stay (days) & $44 \%(86)$ \\
\hline Follow-up (months) & $27 \%(53)$ \\
\hline Lost to follow-up before confirming union & $22 \%(44)$ \\
\hline
\end{tabular}

Non-missing data is reported as medians (interquartile ranges) or as frequencies, with counts indicated in parentheses. ICU: intensive care unit
Bony union was achieved in 179 patients (91\%) after the initial fixation. A total of 21 patients (11\%) in the entire cohort developed FRIs (Table IV). No associations between demographic or treatment-related risk factors and treatment outcomes were observed (Table $\mathrm{V}$ ).

All definitive fixation procedures showed high proportions of union, with circular external fixators having the lowest union rate (86\%). Similarly, the presence of FRI was highest in the circular fixation group (21\%) (Table $V I)$.

\section{Discussion}

The first finding of this study was the demographic sample and the predominant involvement of young males ( $89 \%$ male with a mean age of $29.2 \pm 10.2$ years), which is similar to previous published reports on orthopaedic injuries following civilian gunshots. ${ }^{3,6,8}$ A recent study conducted in South Africa reported a higher rate of intentional and gang-related violence together with the use of alcohol and illegal drugs among young, gunshot-injured males. ${ }^{5}$ While we did not test patients for alcohol or illegal drugs in the present study, our finding, together with the previous reports, again highlights this group of individuals to be a potential target for intervention in the future.

The second important finding relates to our individualised approach to fracture management that produced an overall union

Table IV: Treatment outcomes of included patients

\begin{tabular}{|l|c|}
\hline & $\mathbf{n = 1 9 7}$ \\
\hline Fracture healing & \\
\hline Union & $91 \%(179)$ \\
\hline Non-union & $7 \%(13)$ \\
\hline Unknown & $3 \%(5)$ \\
\hline Fracture-related infection & \\
\hline No & $87 \%(172)$ \\
\hline Yes & $11 \%(21)$ \\
\hline Unknown & $2 \%(4)$ \\
\hline
\end{tabular}

Non-missing data is reported as medians (interquartile ranges) or frequencies, with counts indicated in parentheses. Outcomes of patients that were lost to follow-up are reported as 'unknown'

Table V: Demographic-, clinical- and treatment-specific risk factors for non-union and infection

\begin{tabular}{|c|c|c|c|c|c|c|}
\hline Risk factor & Union* $(n=179)$ & Non-union* $(n=13)$ & p-value & No infection ${ }^{* *}(n=172)$ & Infection $^{* *}(n=21)$ & p-value \\
\hline \multicolumn{7}{|l|}{ Patient demographics } \\
\hline Age (years) & $28.4 \pm 10.4(179)$ & $32.7 \pm 10.1(13)$ & 0.153 & $28.5 \pm 10.1(172)$ & $32.3 \pm 10.0(21)$ & 0.113 \\
\hline Sex (\% male) & $88.3(158)$ & $100.0(13)$ & 0.367 & $87.2(150)$ & $100.0(21)$ & 0.139 \\
\hline $\begin{array}{l}\text { HIV status } \\
\% \text { pos } \\
\% \text { neg } \\
\% \text { unknown }\end{array}$ & $\begin{array}{l}32.4(58) \\
29.6(53) \\
38.0(68)\end{array}$ & $\begin{array}{l}53.9(7) \\
15.4(2) \\
30.8(4)\end{array}$ & 0.263 & $\begin{array}{l}31.4(54) \\
30.8(53) \\
37.8(65)\end{array}$ & $\begin{array}{c}476(10) \\
14.3(3) \\
38.1(8)\end{array}$ & 0.197 \\
\hline \multicolumn{7}{|l|}{ Injury characteristics } \\
\hline $\begin{array}{l}\text { Injury location } \\
\% \text { proximal } \\
\% \text { midshaft } \\
\% \text { distal }\end{array}$ & $\begin{array}{c}7.8(14) \\
91.6(164) \\
0.56(1)\end{array}$ & $\begin{array}{c}0.0(0) \\
92.3(12) \\
7.7(1)\end{array}$ & $>0.999^{\#}$ & $\begin{array}{c}7.6(13) \\
91.9(158) \\
0.6(1)\end{array}$ & $\begin{array}{c}9.5(2) \\
85.7(18) \\
4.8(1)\end{array}$ & $0.406^{\#}$ \\
\hline Intra-articular (\% yes) & $5.0(9)$ & $7.7(1)$ & 0.513 & $5.2(9)$ & $4.8(1)$ & $>0.999$ \\
\hline \multicolumn{7}{|c|}{ Treatment characteristics } \\
\hline Time delay (days) & $2.0(2.0-5.0)(174)$ & $3.0(2.0-5.0)(13)$ & 0.361 & $2.0(2.0-4.0)(166)$ & $3.0(2.0-5.0)(21)$ & 0.457 \\
\hline
\end{tabular}


Table VI: Outcomes related to union and infection of all patients per type of definitive treatment

\begin{tabular}{|c|c|c|}
\hline Definitive procedure & $\begin{array}{l}\text { Union } \\
\% \text { (n) }\end{array}$ & $\begin{array}{c}\text { Infection } \\
\% \text { (n) }\end{array}$ \\
\hline \multicolumn{3}{|c|}{ Debridement and POP (n=86) } \\
\hline Yes & $93 \%(80)$ & $5 \%(4)$ \\
\hline No & $4 \%(3)$ & $92 \%(79)$ \\
\hline Unknown & $4 \%(3)$ & $4 \%(3)$ \\
\hline \multicolumn{3}{|c|}{ Intramedullary nail $(n=53)$} \\
\hline Yes & $91 \%(48)$ & $13 \%(7)$ \\
\hline No & $6 \%(3)$ & $85 \%(45)$ \\
\hline Unknown & $4 \%(2)$ & $2 \%(1)$ \\
\hline \multicolumn{3}{|l|}{ Circular fixator $(n=44)$} \\
\hline Yes & $86 \%(38)$ & $21 \%(9)$ \\
\hline No & $14 \%(6)$ & $80 \%(35)$ \\
\hline \multicolumn{3}{|l|}{ Plate fixation $(n=14)$} \\
\hline Yes & $93 \%(13)$ & $7 \%(1)$ \\
\hline No & $7 \%(1)$ & $93 \%(13)$ \\
\hline
\end{tabular}

Data is presented as frequencies with counts indicated in parentheses. POP: plaster of Paris

rate of $91 \%$ and an $\mathrm{FRI}$ rate of $11 \%$. A recent retrospective study by Metcalf et al. investigated the surgical outcome of tibia fractures managed with intramedullary nail fixation and observed a $30 \%$ nonunion rate and $9 \%$ infection rate for civilian gunshot tibia fractures which was comparable to our findings. ${ }^{17}$ In terms of length of stay, the current study results (median of 6.0 days) were slightly less than that reported by Abghari et al. who investigated the outcome of civilian gunshot injuries and reported a median length of stay of 7.1 days for patients who sustained lower extremity fractures. ${ }^{18}$

There were no associations noted between the demographic or treatment-specific risk factors and the clinical outcomes. No associations between clinical outcome and HIV status were observed. Very few studies have investigated the association between HIV and bone healing and this relationship remains poorly understood and unclear. An ongoing study investigating the association between union and HIV infection is nearing completion, and should provide important information on the effect of HIV on bone healing. ${ }^{19}$

Patients in the present study waited a median of two days for surgery (IQR 2-5 days). This delay is attributed to the high burden of trauma cases at South African hospitals which hampers the ability of providing immediate surgery on admission. ${ }^{3}$ Interestingly, the delay to surgery was not associated with an increased complication rate. Kale et al. had previously reported an infection rate of $11.4 \%$ for patients with open fractures, most commonly arising from motor vehicle accidents, that received wound closure within a week of admission, compared to $77.8 \%$ for patients suffering infection when they had wound closure after two weeks, suggesting an association between delay in surgery and rate of infection. ${ }^{20}$ The lack of an association in the present study could potentially be as a result of the different injury mechanism, faster median time to surgery, or the fact that all surgeries were performed in a single setting, rather than in a staged process. Ultimately, it is important to keep in mind that risk of infection is influenced by multiple factors.

Various definitive skeletal stabilisation procedures were included in this study. Formal debridement followed by cast immobilisation, as well as plate fixation, showed the optimal union and lowest infection rates in the present study. There was no association of $\mathrm{FRI}$ and union rates with length of stay. Minor complications, such as the presence of pressure sores, were not recorded as part of this investigation. Obvious selection bias for cast immobilisation of stable fracture patterns, isolated fractures, low energy transfer injuries and lower grade wounds should, however, be borne in mind when interpreting these results. For this reason, no statistical analysis was performed to investigate associations between treatment modality and outcome, because this relationship would be confounded by the differences in injury characteristics which guides clinical decision-making with regard to treatment modalities. IMN fixation showed outcomes comparable to a previous publication by Hilton et al. who reported a $76.9 \%$ initial union rate following intermedullary nailing for gunshot tibial shaft fractures, with three patients developing osteomyelitis. ${ }^{8}$ The union rate observed in the present study for IMN was $91 \%$.

Circular external fixation was generally reserved for patients who had complex fractures, significant soft tissue damage and delays to surgery, and showed lowest union rate when compared to other definitive fixation methods. A study conducted by Van der Walt and Ferreira however reported higher union rates of $100 \%$ using circular external fixators in their gunshot-induced tibia fracture sample. ${ }^{21}$ The lower union rate with the use of circular external fixation observed in the present study may be attributed to the injury characteristics and delay in treatment for patients who were managed with this fixation modality. Furthermore, circular external fixation showed the highest FRI rates when compared to other definitive fixation methods, keeping in mind that circular fixation was reserved for patients with significant soft tissue injury or delay to surgery. FRI rates were higher in the IMN group (13\%) compared to plate fixation (7\%), but it is important to keep in mind that nail fixation was generally reserved for diaphyseal fractures compared to plate fixation, which was typically employed for metaphyseal injuries. Unfortunately, there is very limited clinical data specifically investigating tibial plate fixation following lowenergy gunshot injuries. Sitnik and Beletsky reported an $8.75 \%$ infection rate in plate fixation in an investigation of 80 patients. This series, however, only included a single gunshot wound case and is therefore not directly comparable to the result of the present study, where a $7 \%$ infection rate was observed. ${ }^{22}$ It is again important to emphasise that risk of infection is influenced by multiple factors and as such, future experimental studies, where bias is largely removed, should investigate this finding further.

The major study limitations include the retrospective design and single centre cohort which resulted in, albeit a large cohort compared to previous reports, too small a sample to detect large differences between sub-groups. The short follow-up period reported in this investigation is another limitation: we report a median follow-up time of 4.1 months, while these injuries should ideally be seen over a longer follow-up period. Lost to follow-up is however a notorious problem in our setting that has previously been described by Badenhorst et al., ${ }^{23}$ and we believe that the results of the study are still meaningful, regardless of this limitation. Finally, the observational nature of the study includes an inherent selection bias in the outcomes observed for the different treatment modalities used where some modalities were more likely to be used in more complex fractures than others. Subsequently, we could not comment on the risk of specific outcomes following treatment with specific devices.

\section{Conclusion}

Tibial fractures caused by civilian gunshots remain challenging injuries to manage. This study found that an individualised approach to the treatment of these fractures can produce a satisfactory union rate with minimal complications if managed during a single stage by intramedullary nails, plate fixation or cast immobilisation. While the complications were higher in patients managed with circular 
external fixation, these devices were generally reserved for more severe injuries.

\section{Ethics statement}

The authors declare that this submission is in accordance with the principles laid down by the Responsible Research Publication Position Statements as developed at the 2nd World Conference on Research Integrity in Singapore, 2010.

Ethical approval for this study was obtained from the Stellenbosch University Ethics Committee, S18/07/139.

All procedures were in accordance with the ethical standards of the responsible committee on human experimentation (institutional and national) and with the Helsink Declaration of 1975 , as revised in 2008.

\section{Declaration}

The authors declare authorship of this article and that they have followed sound scientific research practice. This research is original and does not transgress plagiarism policies.

\section{Author contributions}

MG: Study conceptualisation, data capture, data analysis, first draft preparation, manuscript revision and approval of final manuscript

SJ: Data capture and approval of final manuscript $\mathrm{MvH}$ : Data capture and approval of final manuscript NIR: Data capture and approval of final manuscript SvdM: Data capture and approval of final manuscript OM: Data capture and approval of final manuscript GdP: Data capture and approval of final manuscript MB: Data analysis, manuscript revision and approval of final manuscript NF: Study conceptualisation, data analysis, first draft preparation, manuscript revision and approval of final manuscript

\section{ORCID}

Gerafa M https://orcid.org/0000-0002-6028-0759 Jakoet S https://orcid.org/0000-0002-0009-2203 Van Heukelum M https://orcid.org/0000-0001-9160-7796 Le Roux N https://orcid.org/0000-0001-9115-7254 Van der Merwe S https://orcid.org/0000-0001-6892-4582 Makhubalo O https://orcid.org/0000-0003-0085-0876 Burger M 19 https://orcid.org/0000-0003-2831-4960 Ferreira N https://orcid.org/0000-0002-0567-3373

\section{References}

1. Nunes ED. United Nations Office on Drugs and Crime (UNODC). Global study on homicide: trends, context, data. Vienna: UNODC; 2011. Ciência \& Saúde Coletiva. 2012;17:3447-9.

2. Haagsma JA, Graetz N, Bolliger I, et al. The global burden of injury: incidence, mortality, disability-adjusted life years and time trends from the Global Burden of Disease study 2013. Inj Prev. 2016;122:3-18. https://doi.org/10.1136/ injuryprev-2015-041616.

3. Zaidi AA, Dixon J, Lupez K, et al. The burden of trauma at a district hospital in the Western Cape Province of South Africa: prospective observational study. Afr J Emerg Med. 2019:191:4-20. https://doi.org/10.1016/j.afjem.2019.01.007

4. Engelmann EW, Maqungo S, Laubscher M, et al. Epidemiology and injury severity of 294 extremity gunshot wounds in ten months: a report from the Cape Town trauma registry. SA Orthop J. 2019;182:31-6. https://doi. org/10.17159/2309-8309/2019/v18n2a3.

5. Martin C, Thiart G, McCollum G, Roche S, Maqungo S. The burden of gunshot injuries on orthopaedic healthcare resources in South Africa: retrospective review. S Afr Med J. 2017;107:626-30. https://doi.org/10.7196/SAMJ.2017. v107i7.12257.

6. Jakoet MS, Burger M, Van Heukelum M, et al. The epidemiology and orthopaedic burden of civilian gunshot injuries over a four-year period at a level one trauma unit in Cape Town, South Africa: a retrospective review. Int Orthop. 2020;121:8. https://doi.org/10.1007/s00264-020-04723-6.

7. Sathiyakumar V, Thakore RV, Stinner DJ, et al. Gunshot-induced fractures of the extremities: a review of antibiotic and debridement practices. Curr Rev Musculoskelet Med. 2015;182:76-89. https://doi.org/10.1007/ s12178-015-9284-9.

8. Hilton TL, Kruger N, Wiese KR, Martin CW, Maqungo S. Gunshot tibia fractures treated with intramedullary nailing: A single centre retrospective review. SA Orthop J. 2017;163:2-8. https://doi.org/10.17159/2309-8309/2017/v16n1a4

9. Cross III WW, Swiontkowski MF. Treatment principles in the management of open fractures. Indian J Orthop. 2008;37-7. https://doi. org/10.4103/0019-5413.43373
10. Fang $X$, Jiang $L$, Wang $Y$, Zhao $L$. Treatment of Gustilo grade III tibial fractures with unreamed intramedullary nailing versus external fixator: a meta-analysis. Med Sci Monit. 2012;18(4):49-56. https://doi.org/10.12659/msm.882610.

11. Su CA, Nguyen MP, O'Donnell JA, Vallier HA. Outcomes of tibia shaft fractures caused by low energy gunshot wounds. Injury. 2018;49(7):1348-52. https://doi. org/10.1016/j.injury.2018.05.006.

12. Hardcastle T. The $11 \mathrm{P}$ 's of an Afrocentric trauma system for South Africa: time for action. S Afr Med J. 2011;101(3):160-2. https://doi.org/10.7196/SAMJ.4578.

13. Müller ME, Nazarian $S$, Koch P. The comprehensive classification of fractures of long bones. Springer-Verlag: Berlin; 1990.

14. Papasoulis E, Patzakis MJ, Zalavras CG. Antibiotics in the treatment of low-velocity gunshot-induced fractures: a systematic literature review. Clin Orthop Relat Res. 2013;471-12:3937-44. https://doi.org/10.1007/ s11999-013-2884-z.

15. Whelan DB, Bhandari $M$, Stephen $\mathrm{D}$, et al. Development of the radiographic union score for tibial fractures for the assessment of tibial fracture healing after intramedullary fixation: retrospective review. J Trauma Acute Care Surg 2010;168:629-32. https://doi.org/10.1097/TA.0b013e3181a7c16d.

16. Metsemakers WJ, Morgenstern M, McNally MA, et al. Fracture related infection: a consensus on definition from an international expert group. Injury. 2018;149:505-10. https://doi.org/10.1016/j.injury.2017.08.040.

17. Metcalf KB, Smith EJ, Wetzel RJ, Sontich JK, Ochenjele G. Comparison of clinical outcomes after intramedullary fixation of tibia fractures caused by blunt trauma and civilian gunshot wounds: a retrospective review. J Orthop Trauma. 2020;134:208-13. https://doi.org/10.1097/BOT.000000000000170.

18. Abghari M, Monroy A, Schubl S, Davidovitch R, Egol K. Outcomes following low-energy civilian gunshot wound trauma to the lower extremities: results of a standard protocol at an urban trauma center. lowa Orthop J. 2015;35:65-9.

19. Graham SM, Harrison WJ, Lalloo DG, et al. HOST Study-HIV in Orthopaedic Skeletal Trauma Study: protocol for a multicentre case-cohort study. SA Orthop J. 2018:17:53-8. https://doi.org/10.17159/2309-8309/2018/v17n3a7.

20. Kale AR, Sonawane CS, Waghmare VU, Kalambe H. Open fractures and incidence of infection in tertiary care government hospital. Int J Sci Stud. 2017;5(5):24-8. https://doi.org/10.17354/ijss/2017/386.

21. Van der Walt N, Ferreira N. An audit of circular external fixation usage in a tertiary hospital in South Africa: retrospective review. SA Orthop J. 2018;17:1421. https://doi.org/10.17159/2309-8309/2018/v17n4a1.

22. Sitnik AA, Beletsky AV. Minimally invasive percutaneous plate fixation of tibia fractures results in 80 patients: retrospective review. Clin Orthop Relat Res. 2013;1471:2783-9. https://doi.org/10.1007/s11999-013-2841.

23. Badenhorst DH, Van der Westhuizen CA, Britz E, Burger MC, Ferreira N. Los to follow-up. Challenges to conducting orthopaedic research in South Africa: retrospective review. S Afr Med J. 2018;108:917-21 https://doi.org/10.7196/ SAMJ.2018.v108i11.13252. 